Aims. A\&E departments are busy places; with quick triage decisions required to prioritise urgent care to those who need it. This requires the use of predictions based on past experiences and probabilities. However, this runs the risk of patients being categorised by the prejudices and stigmas associated with their conditions; particularly in the case of mental health patients and the assumption they are otherwise 'medically fit'. This is especially of concern when considering that mental health often deteriorates during acute physical illness.

Following a number of dangerous 'near misses', this audit was conducted to review the practice of triage and physical assessment of patients presenting to A\&E with mental health symptoms. The aim was to compare practice against the Royal College of Emergency Medicine (RCEM) guidelines, to identify repeated issues and systemic vulnerabilities which endangered patients through a lack of appropriate assessment.

Method. Using the online Electronic Patient Record (EPR) system, the notes of 100 patients referred to the Bolton Mental Health Liaison Team (MHLT) from Bolton A\&E were reviewed. They were assessed for whether or not the patients had been appropriately physically assessed, according to the RCEM guidelines, before being referred to the MHLT. These results were analysed anonymously.

Result. The findings showed that less than half (44\%) of all referred patients had physical observations taken at all, and even fewer (37\%) received the full, physical assessment before referral. Out of the patients identified as having abnormal physical observations only $58 \%$ were acted on. Many patients had no history or triage assessment completed; with triage referrals consisting of only the words "mental health". Most importantly, the audit identified this lack of adequate physical assessment resulted in a $2 \%$ 'near miss' rate, including a missed diabetic ketoacidosis and delayed treatment for a missed overdose.

Conclusion. Following this audit and the above result, it is clear that triage and physical assessment of mental health patients attending $\mathrm{A} \& \mathrm{E}$ is inadequate; with resulting risk of severe consequences to patients. It is therefore recommended to co-develop joint guidelines and teaching to guide A\&E and MHLT practitioners on the process of completing the physical assessment prior to referral. It is also recommended to repeat this audit throughout other hospital trusts, in order to review the local referral pathways to ensure adequate physical assessment to avoid any 'near misses' or serious incidents.

\section{Annual physical health checks within a forensic inpatient service}

Michael Cooper* and Partha Gangopadhyay

NHS Ayrshire \& Arran

${ }^{\star}$ Corresponding author.

doi: 10.1192/bjo.2021.232

Aims. Patients prescribed antipsychotics are at risk of ill effects to their physical health. Our aims were to assess whether inpatients within a forensic service, on antipsychotic medications, were receiving annual physical health monitoring in accordance with current NICE and SIGN Guidelines. Based on these Guidelines the following objectives were identified:

1: Physical examination, BMI and blood pressure recorded within the past year

2: FBC recorded within the past year

3: U\&Es recorded within past year

4: LFTs recorded within the past year
5: HbA1C / random glucose / fasting glucose recorded within the past year

6: Random lipids / fasting lipids recorded within the past year

Method. Inclusion Criteria: Patients admitted for longer than a year currently prescribed an antipsychotic.

Data were collected cross-sectionally on $24 / 7 / 20$ for all inpatients meeting the inclusion criteria. Medical notes and the blood results system were reviewed for results of any annual physical examinations and blood monitoring over the past year.

Anonymized data were analysed using Excel.

Result. 13 out of 17 inpatients fulfilled the inclusion criteria. Of these 13 inpatients, 9 (69.2\%) were prescribed clozapine, 1 (7.7\%) zuclopenthixol, 1 (7.7\%) paliperidone and 1 (7.7\%) amisulpride.

All patients had BMI and blood pressures recorded within the preceding month. Only 1 patient (7.7\%) had an annual physical health examination within the past year.

Findings for bloods taken within the past year were as follows:

12 patients $(92.3 \%)$ had an FBC recorded

9 patients $(69.2 \%)$ had $U+$ Es recorded

9 patients $(69.2 \%)$ had LFTs recorded

11 patients $(84.6 \%)$ had HBAlc recorded

7 patients $(53.8 \%)$ had lipids recorded

Conclusion. There is scope for improvement with both annual physical examinations and blood monitoring.

All patients had regular BMIs and blood pressure recorded which is largely attributable to nursing staff protocols. Low compliance with full annual physical examination could be explained by there being no local system in place for annual physical health checks and also frequent changes in junior doctor ward cover.

Blood monitoring showed variable compliance with established standards. FBC monitoring had the best compliance, likely because the vast majority of our patients are prescribed clozapine, which necessitates minimal monthly FBC monitoring.

This audit was presented to the Forensic Team and thereafter it was agreed for a local system to be put in place for annual physical health checks in the summer each year. This will improve oportunities to optimise our patients health. We plan to re-audit at this time.

\section{Racial representation of psychological services in a London male remand prison}

Josh Covey ${ }^{1 \star}$, Joanne Williams ${ }^{1}$, Radha Kothari ${ }^{2}$ and Scott Bartle ${ }^{1}$ ${ }^{1}$ HMP Wormwood Scrubs and ${ }^{2}$ Barnet, Enfield and Haringey Trust ${ }^{*}$ Corresponding author.

doi: 10.1192/bjo.2021.233

Aims. To investigate whether racial groups are proportionally represented in referrals for trauma, hearing voices, emotional regulation and psychological therapy.

To understand the psychological needs across racial groups in HMP Wormwood Scrubs, the UK's 4th-most diverse prison.

To see if the long-established under-representation of Asian males and over-representation of Mixed males in psychological services in the community is also occuring in the prison system. Method. Psychological referrals were recieved via the medical notes system (SystmOne), whereby a prisoner's name, age, location, racial group and reason for referral are trasnferred into the psychology referrals database.

773 referrals were made between October 2018 and May 2020. As the prison's population throughout this time period was fluid, the month of December 2019 was used as a reference for the general prison poipulation. 